\section{J.-M. WATELET}

INERIS - Direction des risques du sol et du sous-sol $B P n^{\circ} 2$

60550 Verneuil-en-Halatte jean-marc.watelet@ineris.fr

D. MONTAGNE

Service municipal des carrières de Laon

8, rue Sérurier 02000 Laon

\section{WOJNAROWICZ}

SEPIA

26, rue Ampère 91430 Igny be.sepia@free.fr

\title{
Risques d'effondrement
}

de carrières souterraines en milieu urbain. De la prévention à la mise en sécurité : exemple de l'îlot Saint-Julien à Laon (02)

La ville de Laon (département de l'Aisne) dispose, depuis I'automne 2001, d'un Plan de prévention des risques naturels prévisibles (PPR) relatif aux mouvements de terrain. Le risque d'effondrement est lié à la présence de cavités souterraines abandonnées situées dans les calcaires lutétiens constituant l'entablement de la butte de Laon.

Dans le quartier dit « Saint-Julien » situé en plein centreville historique, le Service municipal des carrières a pu constater des désordires à caractère évolutif affectant les bâtiments de surface et les ouvrages souterrains correspondants. Une analyse géotechnique a établi que l'origine de ces désordres est directement imputable à la ruine de galeries creusées dans les niveaux de sables glauconieux constituant l'assise des bancs calcaires. Il convient donc, pour assurer la sécurité des personnes et des biens en surface, de procéder au traitement du sous-sol en privilégiant le remblayage de ces cavités à l'origine des dẻsordres. Ces travaux présentent cependant un caractère technique particulier lié à l'environnement urbain mais surtout à l'existence dans les galeries à traiter d'une couche épaisse de matière organique anthropique (ces galeries ayant servi jusqu'à récemment de fosses septiques) plus ou moins solidifiée et probablement très compressible.

Plusieurs solutions ont été étudiées pour mettre en cuvre une consolidation des galeries par injection de béton en plusieurs phases, en considérant que la couche d'excréments possède une capacité de consolidation comparable à une tourbe. Dans un deuxième temps, les galeries des niveaux supérieurs pourront, en fonction de leur état de dégradation, soit être remblayées à l'aide de mousse de résine expansive, soit confortées (maçonneries, cerclages...), de façon à être réutilisées.

Mots-clés : cavités souterraines, plan de prévention des risques, effondrement, galeries superposées, remblayage. 


\section{Risk of underground cavities collapse in urban areas - from prevention to securiting: the case study of the îlot St-Julien in Laon (France, 02)}

Since the autumn 2001 the city of Laon has of a Plan of Prevention of the Predictable Natural Risks concerning potential ground instabilities. The risk of collapse is connected to the presence of abandoned underground cavities located in lutetian limestones constituting the upper hard level of Laon's hillock. In the "St-Julien $x$ district, the historical town centre, the Municipal Service of the Careers noticed disorders with evolutionary character affecting the buildings at ground level and the corresponding undercround works. A geotechnical analysis established that the origin of these disorders is directiy linked with the failure of galleries excavated in the levels of glauconian sands situated at the base of the calcareous benches. In order to garanty the security of people and structures in surface, one has to proceed to the treatment of the subsoil by privileging the backfilling of the cavities at the origin of the disorders. However these works take on a particular technical character because of the urban environment, especially the presence in galleries to be treated of a thick layer of anthropological organic matter (these galleries having been used until recently as septic tanks) more or less solidified and probably very compressible.

Several solutions were studied to operate a consolidation of galleries by concrete injection in several phases, by considering that the layer of excrements has a capacity of consolidation comparable to a peat. Then, the galleries of the upper levels can. according to their state of degradation, either be backfilled with expansive resin foam, or consolidated (masonries, hoopings...). so as to be reused.

Key words : underground cavities, collapse hazard evaluation. multi-level galeries, backfill.

\section{Contexte général}

La ville de Laon (département de l'Aisne) se caractérise par un centre historique de 77 hectares, bâti sur une butte témoin résultant du dégagement par l'érosion des formations tertiaires du Bassin parisien.

Pour des raisons géologiques et historiques, le soussol de la ville haute, cœur économique politique et religieux de la cité médiévale, a été longtemps exploité pour permettre l'édification de bâtiments sur le plateau et pour renforcer localement le réseau défensif du site.

L'existence de ces vides souterrains rend cette zone urbaine sensible aux mouvements de terrain, et plusieurs effondrements de carrières se sont d'ailleurs produits ces dernières années sur le plateau, mettant en péril les personnes et les biens situés dans leur

\section{Environnement géologique}

Le sommet de la butte, qui domine la plaine crayeuse au nord de 85 mètres, est constitué par un entablement calcaire du Lutétien inférieur et moyen, reposant sur les sables et argiles de l'Yprésien et du Thanétien.

Sous une couverture de remblais anthropiques. dont l'épaisseur varie localement de manière sensible, les formations géologiques du Lutétien sont représentées du sommet vers le bas par :

- des bancs calcaires relativement homogènes sur une dizaine de mètres d'épaisseur, plus sableux et glauconieux vers le bas et présentant à la base des niveaux décimétriques très indurés;

- une couche d'environ deux mètres de sables glauconieux grossiers du Lutétien inférieur avec des passages gréseux.

Ces formations surmontent une couche argileuse 
épaisse (5 à $7 \mathrm{~m}$ ) et résistante, connue sous le nom "d'argile de Laon » (Yprésien supérieur).

Sur le plan hydrogéologique, l'argile de Laon forme un mur imperméable au-dessus duquel s'accumulent les eaux d'infiltration issues des précipitations naturelles et des rejets non contrôlés sur la butte. Les écoulements et le niveau piézométrique de cette nappe perchée restent cependant assez mal connus. Même si ces rejets existaient dès l'origine de l'activité humaine sur le plateau, l'augmentation du volume et l'agressivité des eaux déversées peuvent constituer aujourd'hui une réelle menace pour la stabilité des carrières et des versants de la butte. Ces rejets, ainsi que les fuites qui affectent les différents réseaux, peu- vent engendrer d'importantes fluctuations dans le niveau piézométrique de cette nappe et amplifier l'érosion régressive affectant les cavités creusées dans les sables.

\section{2}

\section{Configuration des cavités souterraines}

Les cavités se développent sur plusieurs niveaux (Fig. 1) qui s'enchevêtrent avec les caves et d'anciens souterrains à usage militaire dans un environnement géotechnique de sub-surface particulièrement modifié par le développement historique de cette ville.

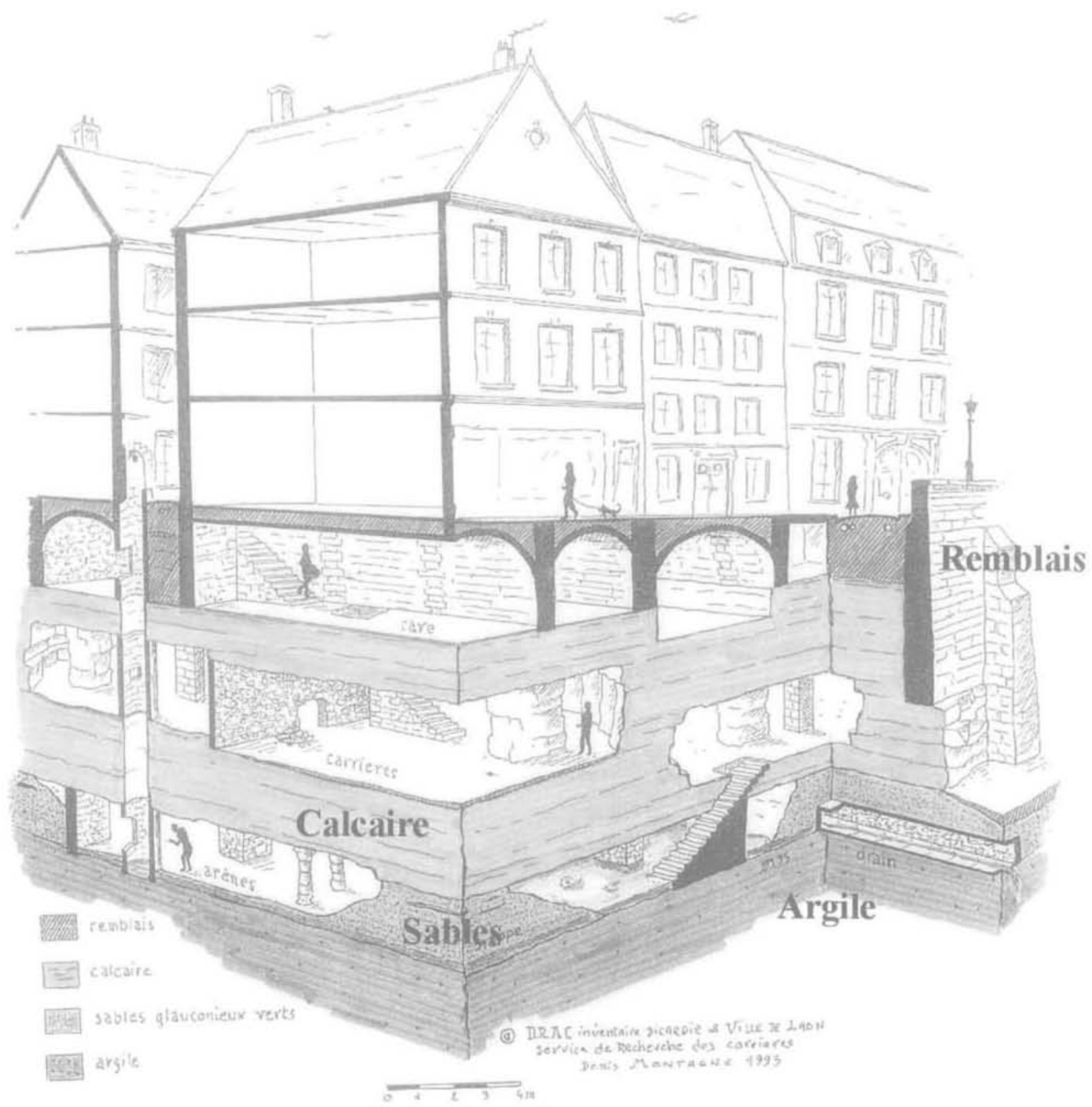

FIG.1 Configuration du sous-sol de Laon.

Laon underground situation. 
Les caves constituent l'assise des bâtiments et sont construites en briques ou en pierres selon différentes architectures et parfois sur plusieurs niveaux. Elles se situent au niveau des remblais et sont souvent fondées sur les bancs calcaires. Elles peuvent s'imbriquer avec les niveaux supérieurs des carrières souterraines et représentent souvent le seul accès possible vers ces cavités.

Les carrières dans les bancs calcaires ont pu servir en partie à l'extraction de matériau pour la construction d'habitations. L'exploitation y a été pratiquée selon la méthode des piliers tournés disposés de manière désordonnée et l'ouverture des galeries avoisine $2 \mathrm{~m}$. L'apparition d'instabilités dans les galeries et l'étroite interaction avec les niveaux de caves et les fondations des maisons ont conduit à l'édification, au cours des âges, de nombreux confortements, en pierre sêche ou en brique. Ces renforcements ont modifié sensiblement l'aspect et la structure des ouvrages souterrains.

Les galeries dans les sables se sont développées de façon désordonnée dans les niveaux de sable glauconieux au-dessus de l'argile de Laon. La plupart sont accessibles à partir de puits verticaux ou d'escaliers venant des carrières et caves supérieures. Le niveau de l'eau dans ces galeries a fortement varié au cours du temps.

\section{3}

\section{Gestion générale du risque}

Depuis l'automne 2001, il existe á Laon un Plan de prévention des risques naturels prévisibles (PPRN) relatif aux mouvements de terrain qui a permis de définir, de hiérarchiser et de cartographier sur le plateau l'aléa lié aux phénomènes d'effondrement de cavités souterraines abandonnées.

Le zonage réglementaire distingue les secteurs urbanisés situés dans l'emprise directe de cavités connues (à l'aplomb et dans la marge de sécurité) et ceux potentiellement sous-minés par des cavités non encore répertoriées.

Dans les secteurs situés au-dessus de zones sousminées répertoriées, le règlement correspondant établi par la préfecture prescrit pour les biens et activités existants un certain nombre de dispositions, dont un examen des cavités accessibles par le Service municipal des carrières.

De plus, pour valoriser la connaissance des risques acquise lors de ce PPRN et favoriser la mise en ceuvre des mesures de protection adaptées, la municipalité de Laon encourage la constitution d'associations de propriétaires pour définir et réduire, à l'échelle de plusieurs habitations, les risques d'effondrement des carrières sous-minant la surface.

\section{2}

\section{Cas de l'îlot Saint-Julien}

Ainsi, dans le quartier dit Saint-Julien, le Service municipal des carrières a pu accéder à plusieurs niveaux de carrières, plus ou moins bien superposés, et a constaté des désordres à caractère évolutif affectant les ouvrages souterrains et les bâtiments de surface correspondants.

\section{Caractéristiques locales du site}

Les parcelles concernées de l'îlot Saint-Julien s'étendent sur environ $1500 \mathrm{~m}^{2}$ dans le centre historique entre une rue commerçante et le rempart sud. Les maisons sont accolées et les cours attenantes sont séparées par de hauts murs anciens. Une petite rue pavée, propriété de la ville, borde ces propriétés privées.

Le premier niveau de carrière s'étend sous la quasitotalité des habitations de I'îlot (une dizaine de parcelles), mais l'alignement d'origine des galeries ne correspond pas tout à fait avec le parcellaire actuel de surface. Les limites de propriété sont d'ailleurs souvent matèrialisées dans les cavités par des muraillements. L'épaisseur de la formation calcaire étant suffisante, on trouve localement un second niveau de carrière sousjacent avec une plus faible ouverture.

En l'absence de deuxième niveau dans les carrières de calcaire, le banc intermédiaire est particulièrement épais ( 3 à $4 \mathrm{~m}$ ) et d'apparence massive. La présence d'un second niveau de carrière juste au-dessus des sables ne laisse par contre subsister qu'une épaisseur de quelques décimètres pour la planche intermédiaire (banc séparatif). Au cours des âges, les cavités dans les sables ont été « remblayées » par des déchets anthropiques. L'ouverture d'origine (estimée entre $1.5 \mathrm{~m}$ et $2 \mathrm{~m}$ ) y est réduite à moins de $0,80 \mathrm{~m}$. Ce niveau a été noyé mais, actuellement, l'eau n'affleure plus au-dessus des remblais. Ceux-ci semblent toutefois saturés et sans cohésion.

Il est donc difficile de pénétrer dans ces vides et donc de cartographier avec précision les limites du réseau. Il apparait cependant nettement que ces vides communiquent entre eux et s'étendent sous plusieurs parcelles. Il est possible également de trouver d'autres cavités isolées dans les sables sans communication avec les galeries situées sous les parcelles adjacentes. Les piliers maçonnés édifiés dans ces carrières reposent sur l'argile de Laon, invisible sous l'épaisse couche de déchets anthropiques, avec des assises qui sont probablement encore baignées par la nappe perchée du lutétien.

\section{2}

\section{Analyse géotechnique des désordres}

Les risques liés à la présence de cavités souterraines d'origine anthropique situées à faible profondeur ont déjà fait l'objet de nombreuses analyses globales (Bonvallet et Chambon, 1980 ; Hoek \& Brown, 1980). Les désordres en carrière résultent souvent de mécanismes classiques de rupture du toit et/ou des piliers (Jeremic, 1987), mais ils peuvent également être induits par des configurations localement complexes et défavorables (Panet et Ricard, 1976; Tritsch et Didier, 1995), comme la présence de plusieurs niveaux oủ les appuis sont mal superposés. La ruine puis l'écroulement d'une cavité sous-jacente engendrent la déconsolidation des ouvrages souterrains dans les niveaux supérieurs et des terrains de recouvrement. A terme, ces mécanismes peuvent provoquer un affaissement en surface, voire un phénomène d'effondrement brutal. L'importance des dommages varie alors selon l'extension du vide 


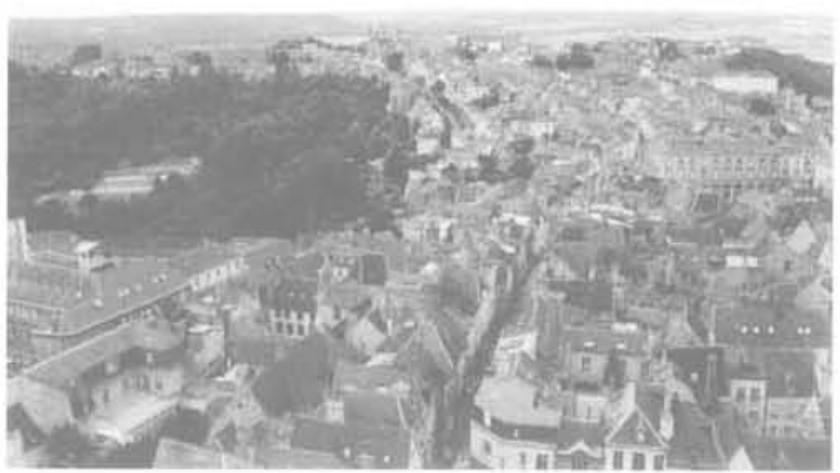

FG. 2 Configuration de l'espace urbain dans la ville haute de Laon.

Laon upper town urbanisation.

affecté, l'épaisseur des remblais et la présence d’une infrastructure sensible en surface du sol.

Sur le site de l'îlot Saint-Julien, la fissuration d'origine mécanique observée dans les caves et les carrières de calcaire affecte les confortements artificiels et le massif rocheux sur les piliers, le toit et même une partie des fronts de masse. Les fractures en traction sur les parements traduisent un déplacement général des structures vers le bas et on note des décollements centimétriques entre le toit et certains piliers naturels (Fig. 3)

Les appuis (piliers naturels et artificiels) des niveaux supérieurs ne remplissent plus leur róle de soutien et la stabilité des terrains en surface est alors conditionnée par la tenue en flexion des bancs calcaires susjacents, lesquels sont déjà naturellement fracturés et hétérogènes.

L'examen géotechnique confirme que le déficit général de portance se situe au niveau de la partie la plus basse dans les galeries de sable. La plupart des piliers maçonnés visibles y sont fortement dégradés, voir ruinés. Le banc de toit immédiat dans ces galeries apparaît très fracturé et présente localement des chutes de toit de plusieurs décimètres d'épaisseur.

On note, en outre, au droit de la carrière de calcaire de deuxième niveau, une rupture du banc intercalaire due au poinçonnement (Fig. 4) d'un pilier artificiel situé dans la galerie de sable, phénomène qui amplifie les désordres à l'échelle du site.

L'origine des désordres peut donc être imputée à la ruine des carrières de sable et au développement consécutif des dégradations dans les niveaux de carrières de calcaire (Fig. 5). Les principaux indices d'affaissement en surface semblent d'ailleurs limités au droit et en bordure immédiate de la zone reconnue comme sous-minée par les galeries dans les sables soit environ $500 \mathrm{~m}^{2}$.

Il faut enfin souligner que ces mécanismes de dégradation présentent un caractère évolutif marqué, notamment dans le deuxième niveau de carrière de calcaire. De plus, ils sont susceptibles d'être aggravés par des facteurs comme la présence non maîtrisée d'eau dans les terrains, de fortes vibrations en surface (trafic routier) ou des travaux d'aménagement en souterrain mal conçus.

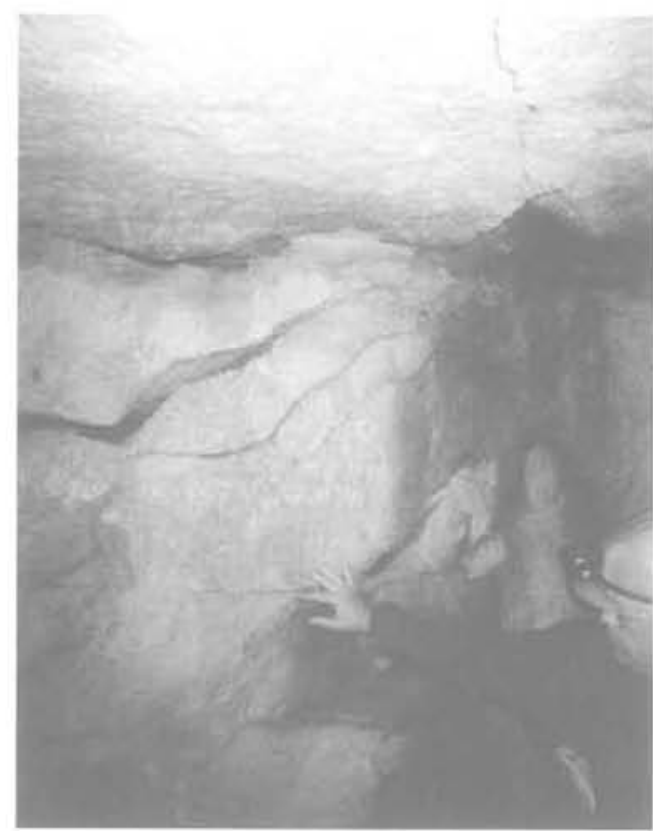

FG.3 Rupture en traction d'un pilier. Tensile fallure of a pillar.

\section{Évaluation des solutions de traitement}

Il convient, pour assurer la sécurité des personnes et des biens (Synthèse de Nainville, 1995; Tritsch, 1995), de procéder rapidement au traitement des cavitès en prenant en compte le problème à l'échelle de l'îlot. Il ne serait, en effet, pas judicieux de mettre en œuvre, ponctuellement dans une ou deux propriétés, des confortements dans les cavités ou sur les maisons sans avoir procédé préalablement au traitement par remblayage des galeries dans les sables qui s'étendent sous tout le site.

Ces travaux revêtent toutefois un caractère technique particulier (AFTES, 1988), lié notamment :

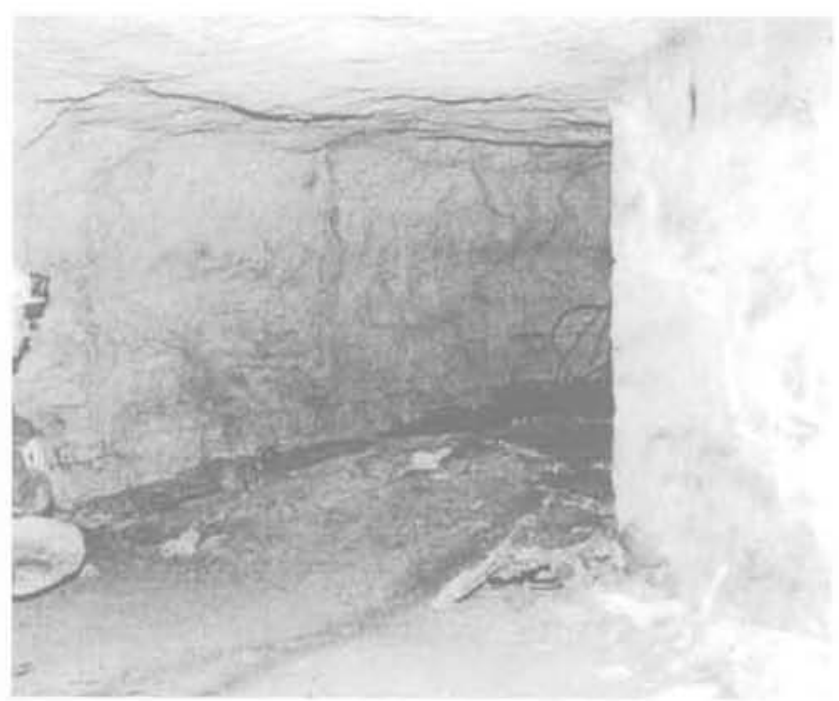

FG.4 Poinçonnement d'un pilier artificiel de galerie de sable au travers de la planche intermédiaire.

Intermediaire layer failure of a pillar in a gallery in sand. 


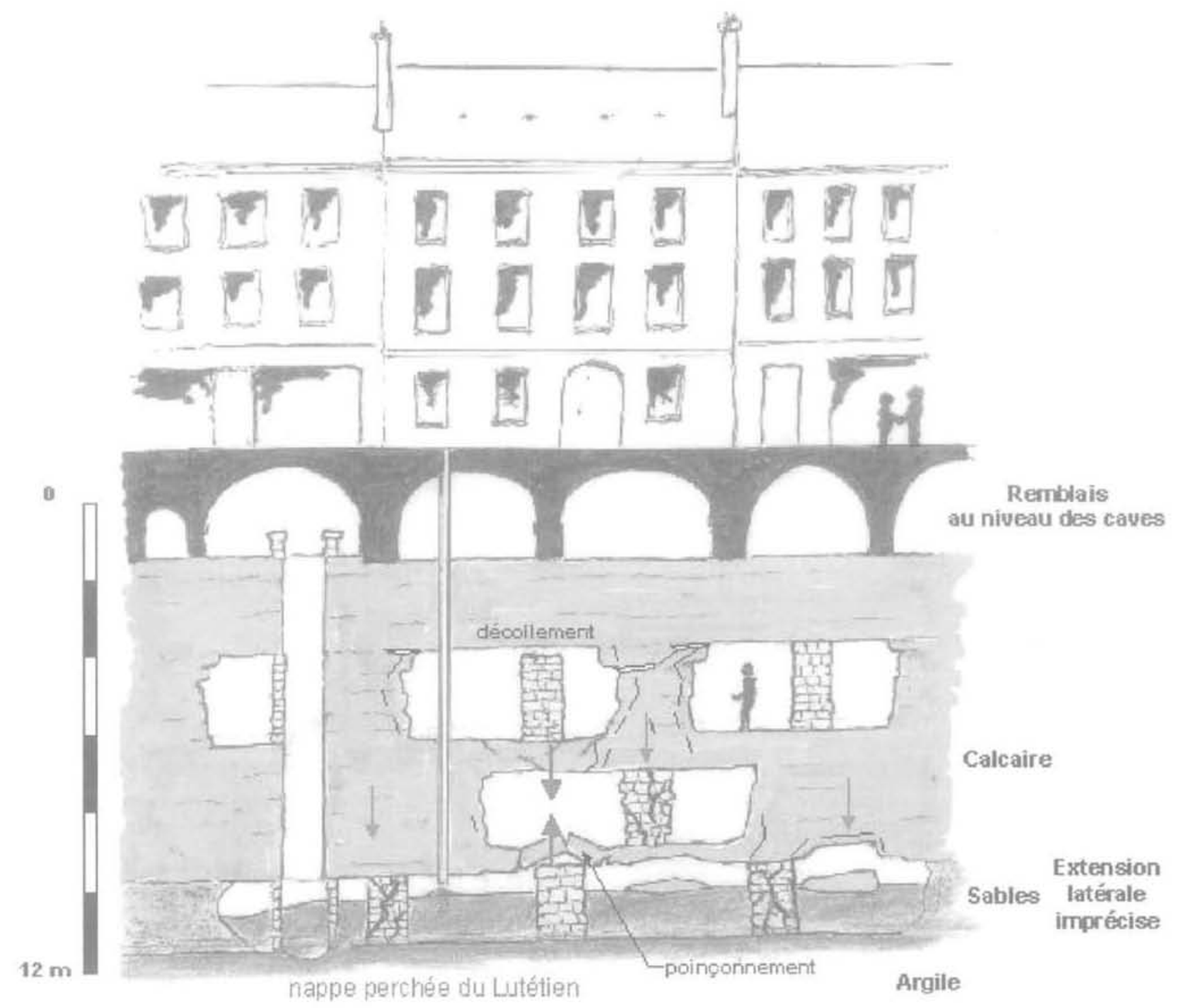

FG. S Mécanisme général de rupture sur le site de l'îlot St-Julien.

Global failure mechanism in St-Julien caves.

- à l'existence, dans les galeries à traiter, d'une couche épaisse de matière organique plus ou moins solidifiée mais probablement très compressible:

- à la connaissance actuellement imparfaite des contours et aux difficultés d'accessibilité à ces vides

- aux risques de dégagement de biogaz $\left(\mathrm{CH}_{4}, \mathrm{CO}_{2}\right.$ $\left.\mathrm{H}_{2} \mathrm{~S} \ldots\right)$ en milieu confiné pendant la phase chantier; - à la présence, en surface, de maisons anciennes et des rues étroites rendant difficile l'amenée de machines.

Compte tenu de ces objectifs et des inconvénients inhérents au site, plusieurs solutions pratiques ont été étudiées pour procéder au remblayage du niveau inférieur de galeries. La stratégie se résume en fait entre une intervention par le fond et une consolidation des galeries par injection de béton depuis la surface.

La première solution suppose de mettre en œuvre des équipes très spécialisées de puisatiers qui devront pénétrer progressivement dans les galeries de sable, dégager par plots la couche de matière organique pour établir des consolidations reposant sur l'argile de Laon et susceptibles de servir de "semelle » aux terrains d'injection pourraient intervenir par la suite (AFTES. 1988; Trisch, 1995).

Les travaux de remblayage sans intervention directe au fond nécessitent de procéder en plusieurs phases, en considérant que la couche organique possède une capacité de consolidation comparable à une tourbe. Le béton injecté peut être mis en place par passes successives, à l'aide d'une pompe et d'une aiguille. L'étude du contexte géotechnique et la prise en compte des conditions de sécurité d'exécution ont permis d'orienter les travaux sur la solution de comblement du niveau inférieur par la méthode d'injection du béton ( autoplaçant $»$. Cette méthode consiste à " pousser $)$ le béton à l'avancement depuis les orifices sans exposer le personnel aux risques d'effondrement.

La résistance du béton ne pourra être déterminée que lors des études d'exécution. L'espace entre le béton qui aura tassé après sa mise en place et le toit des galeries sera rempli gravitairement par des injections puis par clavage depuis la surface.

Ces travaux permettront de stabiliser la base des galeries dans les sables. Ensuite seulement, il est envi- 
sageable de traiter les galeries des niveaux supérieurs qui pourront, en fonction de leur état de dégradation, soit être remblayées en utilisant des mousses expansives d'une résistance de l'ordre de $1 \mathrm{MPa}$, soit être confortées (maçonneries, cerclages...) de façon à être réutilisées.

Après la réalisation et contrôle des travaux de mise en sécurité, des mesures de surveillance devront être mises en ceuvre et se poursuivre pour vérifier la stabilisation du site. Ces mesures peuvent comprendre des inspections visuelles dans les cavités non remblayées avec pose et lecture de témoins.

\section{Difficultés de mise en œuvre}

A ce stade du projet, il apparait que certaines informations comme la précision des contours des galeries dans les sables ou l'accessibilité sur le site, que ce soit au fond ou depuis la surface, vont manquer pour orienter les options techniques et pour préciser les coûts correspondants.

Le choix des actions de mise en sécurité doit également tenir compte du coút global de l'opération, lequel sera supporté en grande partie par des particuliers, au prorata de la surface des parcelles concernées (sans compter les nuisances en phase travaux). Ainsi, on peut considérer que 50 à $60 \%$ des travaux obligatoires dans les galeries de sable seront effectués sous les limites de deux propriétés et $20 \%$ concerneront le domaine public.

Dans ces conditions, il a été établi en première approche que le remblayage par injection depuis le dessus représentait le coút le moins élevé avec un total d'environ 150000 euros HT. Les solutions par le fond constituent des options plus complètes qui permettraient notamment de s'affranchir des incertitudes liées aux contours sur le site, Elles nécessitent cependant des infrastructures plus lourdes pour l'accès aux galeries (puits, tunnel depuis le rempart en contrebas...).

\section{Conclusion et perspectives}

L'opération en cours sur le site de l'îlot Saint-Julien à Laon représente, à divers titres, un exemple original d'opération de mise en sécurité d'un secteur urbain sous-miné par des anciennes carrières abandonnées présentant une configuration complexe.

Dans un premier temps, la présence des cavités sur le site et l'occurrence d'un risque d'effondrement ont pu ètre mises en évidence dans le cadre de la procédure d'un Plan de Prévention des risques naturels "mouvement de terrain ".

Par la suite, la municipalité de Laon a favorisé, par l'intermédiaire de l'action du Service des carrières, l'information et la concertation des riverains pour les inciter à se regrouper en association et pour considérer les solutions de traitement du risque à l'échelle d'un quartier.
Sur le plan technique, la configuration particulière des carrières, s'étendant sur plusieurs niveaux dans des matériaux de nature différente, suppose une analyse géotechnique globale dont les objectif's étaient de déterminer l'origine et l'évolution des mécanismes de rupture et de préciser les priorités pour les traitements. De plus, le niveau de vides le plus profond, à conforter en priorité, est quasiment rempli par des déchets organiques.

Compte tenu des conditions de site en surface (centre historique médiéval classé), de l'état évolutif des caves et des carrières et de la présence de ces déchets compressibles, les travaux de mise en sécurité ne pourront pas s'apparenter à un chantier de comblement classique. Des solutions originales, par le fond ou depuis la surface, ont du être recherchées en considérant les différentes contraintes.

Enfin, le coût global de ces solutions doit être parfaitement réparti entre les différents propriétaires concernés et rester en rapport avec l'évaluation des biens en surface.

A partir des prescriptions établies et de la première évaluation technico-économique, l'association de riverains est maintenant en mesure de rechercher les financements qui lui permettront de lancer la procédure de travaux de mise en sécurité du secteur sousminé et contribuer ainsi à la valorisation future des terrains en surface.

En parallèle, le Service municipal des carrières continue de suivre l'évolution des dégradations et procède à un levé aussi exhaustif que possible des vides pour préciser les contours des vides dans les sables et leur projection en surface.

\section{Bibliographie}

AFIES - a Recommandations relatives aux travaux d'injection

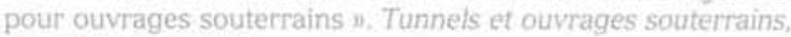
$n^{\circ}$ spécial, mai 1988

Bonvallet.J., Chambon C. - * Détermination d'un coefficient de sécuritè dans les exploitations par chambres et piliers aban. donnés m. Revue francaise de géotechnique, $n^{\circ}$ 13, noy. 1980.

Hoek E., Brown E.T. - Underground Excavation in Rock. Institution of mining and Metallurgy, 1980.

Jeremic M.1. - Ground Mechanics in hard Rock mining. Rotterdam/Brookfield, AA Balkema, 1987.

Panet M. Ricard A. - « Comportement mécanique des massifs rocheux 1. La mécanique des roches appliquée aux ouvrages de génie civil. chap. VI, 1976.

Synthèse des travaux du séminaire de Nainville-les-Roches« Dẻcembre 1993 ; carrières souterraines abandonnées riscues et prévention 1 . Bulletin de l'Association internationale de géologie de l'ingénieur, $n^{\circ}$ 51, 1995.

Tritsch J.J., Didier C. - Guide méthodoloqique pour l'identification et la prévision des mouvements de terrain. Rapport de recherche INERIS pour le compte du ministère de I'Environnement. 1995 .

Tritsch 1.J. - Evvaluation des méthodes et du coút de la mise en sécurité des populations menacées par les risques d'effondrement de carrières. Étude financée par la Direction de la prévention des pollutions et des risques du ministère dé l'Aménagement du territoire et de l'Environnement. 1995. 